\title{
An extension for Rice's integral and applications
}

Tao-Tao Liu and Qiu-Ming Luo*

"Correspondence:

luomath2007@163.com

Department of Mathematics,

Chongqing Normal University,

Chongqing Higher Education Mega

Center, Huxi Campus, Chongqing,

401331, People's Republic of China

\section{Abstract}

In this paper, we generalize Rice's integral and give some applications. Some old and new binomial identities are obtained.

MSC: Primary 05A10; secondary 05A19; 11B65

Keywords: Rice's integral; Cauchy's residue formula; binomial identity

\section{Introduction}

For complex $z$ and $m, n \in \mathbb{N}_{0}=\mathbb{N} \cup\{0\}$, we define that

$$
(z)_{0}=1, \quad(z)_{n}=z(z+1) \cdots(z+n-1)
$$

and

$$
(z+m)_{n-m}= \begin{cases}(m+z)(m+z+1) \cdots(z+n-1), & \text { when } 0 \leq m<n \\ 1, & \text { when } m \geq n\end{cases}
$$

The standard Bell polynomials (see [1, p.133]). The modified Bell polynomials $L_{m}\left(x_{1}\right.$, $\left.x_{2}, \ldots\right)$ are defined by

$$
\exp \left(\sum_{k=1}^{\infty} x_{k} \frac{t^{k}}{k}\right)=1+\sum_{m=1}^{\infty} L_{m}\left(x_{1}, x_{2}, \ldots\right) \frac{t^{m}}{m !}
$$

We have

$$
L_{m}\left(x_{1}, x_{2}, \ldots\right)=\sum_{m_{1}+2 m_{2}+3 m_{3}+\cdots=m} \frac{m !}{m_{1} ! m_{2} ! m_{3} ! \cdots}\left(\frac{x_{1}}{1}\right)^{m_{1}}\left(\frac{x_{2}}{2}\right)^{m_{2}}\left(\frac{x_{3}}{3}\right)^{m_{3}} \cdots
$$

Recalling Rice's integral (see [2] and [3]), suppose that $f(z)$ is a rational function analytic on $\left[0,+\infty\left[\right.\right.$, and $n \in \mathbb{N}_{0}$, then we have

$$
\sum_{k=0}^{n}(-1)^{k}\left(\begin{array}{l}
n \\
k
\end{array}\right) f(k)=\frac{(-1)^{n}}{2 \pi i} \int_{\mathcal{C}} \frac{n !}{z(z-1) \cdots(z-n)} f(z) d z
$$

where $\mathcal{C}$ is a positively oriented, encloses the simple poles at the integers $0,1,2, \ldots, n$ and no others.

@ 2015 Liu and Luo: licensee Springer. This is an Open Access article distributed under the terms of the Creative Commons Attribution License (http://creativecommons.org/licenses/by/4.0), which permits unrestricted use, distribution, and reproduction in any medium, provided the original work is properly credited. 
All technicalities and many examples of Rice's integral are involved in [2] and [3].

Well-known is the following binomial identity: For $n \in \mathbb{N}=\{1,2, \ldots\}$ and $\theta>0$, we have

$$
\sum_{k=0}^{n}(-1)^{k}\left(\begin{array}{l}
n \\
k
\end{array}\right) \frac{\theta}{\theta+k}=\prod_{k=1}^{n} \frac{k}{\theta+k}
$$

Recently, Jonathon [4] gave a simple and interesting probabilistic proof of the above binomial identity, and he further extended this binomial identity based on the probabilistic method. We state his result as follows. For all $r, n \in \mathbb{N} ; \theta>0$, we have

$$
\begin{aligned}
& \sum_{k=0}^{n}(-1)^{k}\left(\begin{array}{l}
n \\
k
\end{array}\right)\left(\frac{\theta}{\theta+k}\right)^{r} \\
& \quad=\left(\prod_{k=1}^{n} \frac{k}{\theta+k}\right)\left(1+\sum_{j=1}^{r-1} \sum_{1 \leq k_{1} \leq \cdots \leq k_{j} \leq n} \frac{\theta^{j}}{\left(\theta+k_{1}\right)\left(\theta+k_{2}\right) \cdots\left(\theta+k_{j}\right)}\right) .
\end{aligned}
$$

More recently, Bayad et al. [5, 6] established new identities for some new polynomials associated with the $\lambda$-array type polynomials. Simsek [6] gave an approach to combinatorial sums involving binomial coefficients and the Catalan number from the analysis of the Bernstein basis functions. There is much literature about the binomial identities, we refer the readers to [7-17].

In the present paper, we further extend Rice's integral by using Cauchy's integral formula. As some applications, some old and new binomial identities are obtained.

\section{Rice's integral}

In this section, we generalize Rice's integral using Cauchy's integral formula.

Theorem 1 Let $f(z)$ be a rational function analytic on $\left[0,+\infty\left[\right.\right.$ and $m, n \in \mathbb{N}_{0}$. Then we have

$$
\sum_{k=0}^{n}(-1)^{k}\left(\begin{array}{l}
n \\
k
\end{array}\right)\left(\begin{array}{l}
n+k \\
m+k
\end{array}\right) f(k)=\frac{(-1)^{n} n !}{2 \pi i(n-m) !} \int_{\mathcal{C}} \frac{(m+z+1)_{n-m}}{z(z-1) \cdots(z-n)} f(z) d z
$$

where $\mathcal{C}$ is a positively oriented, encloses the poles $0,1,2, \ldots, n$ and no others.

Proof By using Cauchy's integral formula, via the residues calculus, we have

$$
\begin{aligned}
& \frac{(-1)^{n} n !}{2 \pi i(n-m) !} \int_{\mathcal{C}} \frac{(z+m+1)_{n-m}}{z(z-1) \cdots(z-n)} f(z) d z \\
& =\frac{(-1)^{n} n !}{(n-m) !} \sum_{k=1}^{n+1} \operatorname{Res}_{z=k-1} \frac{(z+m+1)_{n-m}}{z(z-1) \cdots(z-n)} f(z) \\
& =\frac{(-1)^{n} n !}{(n-m) !} \sum_{k=1}^{n+1} \lim _{z \rightarrow k-1} \frac{(z+m+1)_{n-m}}{z(z-1) \cdots(z-k+2)(z-k) \cdots(z-n)} f(z) \\
& =\frac{(-1)^{n} n !}{(n-m) !} \sum_{k=1}^{n+1} \frac{(m+k)_{n-m}}{(k-1) \cdots 2 \cdot 1(-1)(-2) \cdots(-n+k-1)} f(k-1)
\end{aligned}
$$




$$
\begin{aligned}
& =\frac{n !}{(n-m) !} \sum_{k=0}^{n}(-1)^{k} \frac{(m+k+1)_{n-m}}{k !(n-k) !} f(k) \\
& =\sum_{k=0}^{n}(-1)^{k}\left(\begin{array}{l}
n \\
k
\end{array}\right)\left(\begin{array}{c}
n+k \\
m+k
\end{array}\right) f(k) .
\end{aligned}
$$

The proof is complete.

Corollary 2 Let $f(z)$ be a rational function analytic on $\left[0,+\infty\left[\right.\right.$ and $n \in \mathbb{N}_{0}$. Then we have

$$
\sum_{k=0}^{n}(-1)^{k}\left(\begin{array}{c}
n \\
k
\end{array}\right)\left(\begin{array}{c}
n+k \\
k
\end{array}\right) f(k)=\frac{(-1)^{n}}{2 \pi i} \int_{\mathcal{C}} \frac{(z+1)_{n}}{z(z-1) \cdots(z-n)} f(z) d z
$$

where $\mathcal{C}$ is a positively oriented, encloses the poles $0,1,2, \ldots, n$ and no others.

Proof Taking $m=0$ in Theorem 1, Corollary 2 follows.

Remark 3 Taking $m=n$ in Theorem 1 and noting that $(z+m+1)_{0}=1$, we get Rice's integral (5) immediately. Obviously, Theorem 1 is an extension of Rice's integral (5).

\section{Applications}

In this section, we give some applications of Theorem 1 and Corollary 2. We can obtain many old and new binomial identities.

Below we give an extension of binomial identity (7) and deduce some new binomial identities.

Theorem 4 Let $r, l, n \in \mathbb{N}, m \in \mathbb{N}_{0}, 0 \leq m \leq n ; \theta>0, \beta \neq 0$.

When $\theta \notin\{m+1, m+2, \ldots, n\}$ and $r>0$, we have

$$
\begin{aligned}
\sum_{k=0}^{n}( & -1)^{k}\left(\begin{array}{l}
n \\
k
\end{array}\right)\left(\begin{array}{c}
n+k \\
m+k
\end{array}\right)\left(\frac{\beta}{\theta+k}\right)^{r} \\
= & \left(\prod_{k=1}^{n} \frac{k}{\theta+k}\right)\left(\prod_{k=m+1}^{n} \frac{k-\theta}{k-m}\right) \\
& \times\left(\left(\frac{\beta}{\theta}\right)^{r}+\sum_{j=1}^{r-1} \sum_{1 \leq s_{1} \leq \cdots \leq s_{j} \leq n} \frac{\beta^{r}}{\theta\left(\theta+s_{1}\right)\left(\theta+s_{2}\right) \cdots\left(\theta+s_{j}\right)}\right. \\
& \left.+\sum_{i+l=r-1} \sum_{i=1}^{n-m} \sum_{m+1 \leq k_{1}<\cdots<k_{i} \leq n} \sum_{0 \leq s_{1} \leq \cdots \leq s_{l} \leq n} \frac{\beta^{r}}{\theta\left(k_{1}-\theta\right) \cdots\left(k_{i}-\theta\right)\left(\theta+s_{1}\right) \cdots\left(\theta+s_{l}\right)}\right) .
\end{aligned}
$$

When $\theta \in\{m+1, m+2, \ldots, n\}$ and $r>1$, we have

$$
\begin{gathered}
\sum_{k=0}^{n}(-1)^{k}\left(\begin{array}{l}
n \\
k
\end{array}\right)\left(\begin{array}{l}
n+k \\
m+k
\end{array}\right)\left(\frac{\beta}{\theta+k}\right)^{r} \\
=\frac{1}{(n-m) !}\left(\prod_{k=1}^{n} \frac{k}{\theta+k}\right)
\end{gathered}
$$




$$
\begin{aligned}
& \times\left(\prod_{k=m+1, k \neq \theta}^{n}(k-\theta)\right)\left(\frac{\beta^{r}}{\theta^{r-1}}+\sum_{j=1}^{r-2} \sum_{1 \leq s_{1} \leq \cdots \leq s_{j} \leq n} \frac{\beta^{r}}{\theta\left(\theta+s_{1}\right)\left(\theta+s_{2}\right) \cdots\left(\theta+s_{j}\right)}\right. \\
& \left.+\sum_{i+l=r-2} \sum_{i=1}^{n-m-1} \sum_{\substack{k_{1}, \ldots, k_{i} \neq \theta \\
m+1 \leq k_{1}<\cdots<k_{i} \leq n}} \sum_{0 \leq s_{1} \leq \cdots \leq s_{l} \leq n} \frac{\beta^{r}}{\theta\left(k_{1}-\theta\right) \cdots\left(k_{i}-\theta\right)\left(\theta+s_{1}\right) \cdots\left(\theta+s_{l}\right)}\right) .
\end{aligned}
$$

Proof Taking $f(z)=\left(\frac{\beta}{\theta+z}\right)^{r}$ in (8), and $\mathcal{C}$ is a positively oriented, encloses the poles $0,1,2, \ldots, n$ and no others, by means of the residues calculus, we obtain the following.

- When $\theta \notin\{m+1, m+2, \ldots, n\}$, we have

$$
\begin{aligned}
& \sum_{k=0}^{n}(-1)^{k}\left(\begin{array}{l}
n \\
k
\end{array}\right)\left(\begin{array}{c}
n+k \\
m+k
\end{array}\right)\left(\frac{\beta}{\theta+k}\right)^{r} \\
& =\frac{(-1)^{n} n !}{2 \pi i(n-m) !} \int_{\mathcal{C}} \frac{(m+z+1)_{n-m}}{z(z-1) \cdots(z-n)}\left(\frac{\beta}{\theta+z}\right)^{r} d z \\
& =\frac{(-1)^{n+1} n !}{(n-m) !} \operatorname{Res}_{z=-\theta} \frac{(m+z+1)_{n-m}}{z(z-1) \cdots(z-n)}\left(\frac{\beta}{\theta+z}\right)^{r} \\
& =\frac{(-1)^{n+1} n !}{(n-m) !}\left[(\theta+z)^{r-1}\right] \frac{(z+1+m) \cdots(z+n) \beta^{r}}{z(z-1) \cdots(z-n)} \\
& =\frac{n !}{(n-m) !}\left[z^{r-1}\right]\left(\prod_{k=1}^{n} \frac{1}{\theta+k}\right)\left(\prod_{k=m+1}^{n}(k-\theta)\right) \frac{\left(1+\frac{z}{m+1-\theta}\right) \cdots\left(1+\frac{z}{n-\theta}\right) \beta^{r}}{\theta\left(1-\frac{z}{\theta}\right)\left(1-\frac{z}{\theta+1}\right) \cdots\left(1-\frac{z}{\theta+n}\right)} \\
& =\left(\prod_{k=1}^{n} \frac{k}{\theta+k}\right)\left(\prod_{k=m+1}^{n} \frac{k-\theta}{k-m}\right)\left[z^{r-l-1}\right]\left(1+\sum_{i=1}^{n-m} \sum_{m+1 \leq k_{1}<\cdots<k_{i} \leq n} \frac{z^{i}}{\left(k_{1}-\theta\right) \cdots\left(k_{i}-\theta\right)}\right) \\
& \times \sum_{0 \leq s_{1} \leq \cdots \leq s_{l} \leq n} \frac{\beta^{r}}{\theta\left(\theta+s_{1}\right)\left(\theta+s_{2}\right) \cdots\left(\theta+s_{l}\right)} \\
& =\left(\prod_{k=1}^{n} \frac{k}{\theta+k}\right)\left(\prod_{k=m+1}^{n} \frac{k-\theta}{k-m}\right)\left[z^{r-l-1}\right]\left(1+\sum_{i=1}^{n-m} \sum_{m+1 \leq k_{1}<\cdots<k_{i} \leq n} \frac{z^{i}}{\left(k_{1}-\theta\right) \cdots\left(k_{i}-\theta\right)}\right) \\
& \times\left(\frac{\beta^{r}}{\theta^{l+1}}+\sum_{j=1}^{l} \sum_{1 \leq s_{1} \leq \cdots \leq s_{j} \leq n} \frac{\beta^{r}}{\theta\left(\theta+s_{1}\right)\left(\theta+s_{2}\right) \cdots\left(\theta+s_{j}\right)}\right) \\
& =\left(\prod_{k=1}^{n} \frac{k}{\theta+k}\right)\left(\prod_{k=m+1}^{n} \frac{k-\theta}{k-m}\right)\left(\left(\frac{\beta}{\theta}\right)^{r}+\sum_{j=1}^{r-1} \sum_{1 \leq s_{1} \leq \cdots \leq s_{j} \leq n} \frac{\beta^{r}}{\theta\left(\theta+s_{1}\right)\left(\theta+s_{2}\right) \cdots\left(\theta+s_{j}\right)}\right. \\
& \left.+\sum_{i+l=r-1} \sum_{i=1}^{n-m} \sum_{m+1 \leq k_{1}<\cdots<k_{i} \leq n} \sum_{0 \leq s_{1} \leq \cdots \leq s_{l} \leq n} \frac{\beta^{r}}{\theta\left(k_{1}-\theta\right) \cdots\left(k_{i}-\theta\right)\left(\theta+s_{1}\right) \cdots\left(\theta+s_{l}\right)}\right) .
\end{aligned}
$$

- When $\theta \in\{m+1, m+2, \ldots, n\}$, we have

$$
\begin{aligned}
& \sum_{k=0}^{n}(-1)^{k}\left(\begin{array}{l}
n \\
k
\end{array}\right)\left(\begin{array}{c}
n+k \\
m+k
\end{array}\right)\left(\frac{\beta}{\theta+k}\right)^{r} \\
& \quad=\frac{(-1)^{n} n !}{2 \pi i(n-m) !} \int_{\mathcal{C}} \frac{(m+z+1)_{n-m}}{z(z-1) \cdots(z-n)}\left(\frac{\beta}{\theta+z}\right)^{r} d z
\end{aligned}
$$




$$
\begin{aligned}
& =\frac{(-1)^{n+1} n !}{(n-m) !} \operatorname{Res}_{z=-\theta} \frac{(m+z+1)_{n-m}}{z(z-1) \cdots(z-n)}\left(\frac{\beta}{\theta+z}\right)^{r} \\
& =\frac{(-1)^{n+1} n !}{(n-m) !} \operatorname{Res}_{z=-\theta} \frac{(z+1+m) \cdots(z+\theta-1)(z+\theta+1) \cdots(z+n-\theta)}{z(z-1) \cdots(z-n)} \frac{\beta^{r}}{(\theta+z)^{r-1}} \\
& =\frac{(-1)^{n+1} n !}{(n-m) !}\left[(\theta+z)^{r-2}\right] \frac{(z+1+m) \cdots(z+\theta-1)(z+\theta+1) \cdots(z+n-\theta) \beta^{r}}{z(z-1) \cdots(z-n)} \\
& =\frac{(-1)^{n+1} n !}{(n-m) !}\left[z^{r-2}\right] \frac{(z+1+m-\theta) \cdots(z-1)(z+1) \cdots(z+n-\theta) \beta^{r}}{(z-\theta)(z-\theta-1) \cdots(z-n-\theta)} \\
& =\frac{n !}{(n-m) !}\left[z^{r-2}\right]\left(\prod_{k=1}^{n} \frac{1}{\theta+k}\right)\left(\prod_{k=m+1, k \neq \theta}^{n}(k-\theta)\right) \\
& \times \frac{\left(1+\frac{z}{m+1-\theta}\right) \cdots(1-z)(1+z) \cdots\left(1+\frac{z}{n-\theta}\right) \beta^{r}}{\theta\left(1-\frac{z}{\theta}\right)\left(1-\frac{z}{\theta+1}\right) \cdots\left(1-\frac{z}{\theta+n}\right)} \\
& =\frac{1}{(n-m) !}\left(\prod_{k=1}^{n} \frac{k}{\theta+k}\right)\left(\prod_{k=m+1, k \neq \theta}^{n}(k-\theta)\right)\left[z^{r-l-2}\right] \\
& \times\left(1+\sum_{i=1}^{n-m-1} \sum_{\substack{k_{1}, \ldots, k_{i} \neq \theta \\
m+1 \leq k_{1}<\cdots<k_{i} \leq n}} \frac{z^{i}}{\left(k_{1}-\theta\right) \cdots\left(k_{i}-\theta\right)}\right) \\
& \times \sum_{0 \leq s_{1} \leq \cdots \leq s_{l} \leq n} \frac{\beta^{r}}{\theta\left(\theta+s_{1}\right)\left(\theta+s_{2}\right) \cdots\left(\theta+s_{l}\right)} \\
& =\frac{1}{(n-m) !}\left(\prod_{k=1}^{n} \frac{k}{\theta+k}\right)\left(\prod_{k=m+1, k \neq \theta}^{n}(k-\theta)\right)\left[z^{r-l-2}\right] \\
& \times\left(1+\sum_{i=1}^{n-m-1} \sum_{\substack{k_{1}, \ldots, k_{i} \neq \theta \\
m+1 \leq k_{1}<\cdots<k_{i} \leq n}} \frac{z^{i}}{\left(k_{1}-\theta\right) \cdots\left(k_{i}-\theta\right)}\right) \\
& \times\left(\frac{\beta^{r}}{\theta^{l+1}}+\sum_{j=1}^{l} \sum_{1 \leq s_{1} \leq \cdots \leq s_{j} \leq n} \frac{\beta^{r}}{\theta\left(\theta+s_{1}\right)\left(\theta+s_{2}\right) \cdots\left(\theta+s_{j}\right)}\right) \\
& =\frac{1}{(n-m) !}\left(\prod_{k=1}^{n} \frac{k}{\theta+k}\right)\left(\prod_{k=m+1, k \neq \theta}^{n}(k-\theta)\right) \\
& \times\left(\frac{\beta^{r}}{\theta^{r-1}}+\sum_{j=1}^{r-2} \sum_{1 \leq s_{1} \leq \cdots \leq s_{j} \leq n} \frac{\beta^{r}}{\theta\left(\theta+s_{1}\right)\left(\theta+s_{2}\right) \cdots\left(\theta+s_{j}\right)}\right.
\end{aligned}
$$

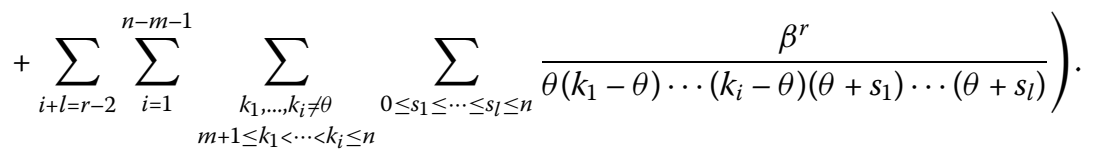

This proof is complete.

Setting $r=1$ and $\theta=\beta$ in (10) of Theorem 4 and $r=2$ and $\theta=\beta$ in (11) of Theorem 4, we have the following new binomial identity.

Corollary 5 Let $n \in \mathbb{N}, m \in \mathbb{N}_{0}, 0 \leq m \leq n ; \theta>0$. 
When $\theta \notin\{m+1, m+2, \ldots, n\}$, we have

$$
\sum_{k=0}^{n}(-1)^{k}\left(\begin{array}{l}
n \\
k
\end{array}\right)\left(\begin{array}{l}
n+k \\
m+k
\end{array}\right) \frac{\theta}{\theta+k}=\left(\prod_{k=1}^{n} \frac{k}{\theta+k}\right)\left(\prod_{k=m+1}^{n} \frac{k-\theta}{k-m}\right) .
$$

When $\theta \in\{m+1, m+2, \ldots, n\}$, we have

$$
\begin{aligned}
\sum_{k=0}^{n}(-1)^{k}\left(\begin{array}{l}
n \\
k
\end{array}\right)\left(\begin{array}{c}
n+k \\
m+k
\end{array}\right)\left(\frac{\theta}{\theta+k}\right)^{2} \\
\quad=\frac{\theta}{(n-m) !}\left(\prod_{k=1}^{n} \frac{k}{\theta+k}\right)\left(\prod_{k=m+1, k \neq \theta}^{n}(k-\theta)\right) .
\end{aligned}
$$

Corollary 6 (see [4]) For $r, n \in \mathbb{N} ; \theta>0$, we have

$$
\begin{aligned}
& \sum_{k=0}^{n}(-1)^{k}\left(\begin{array}{l}
n \\
k
\end{array}\right)\left(\frac{\theta}{\theta+k}\right)^{r} \\
& \quad=\left(\prod_{k=1}^{n} \frac{k}{\theta+k}\right)\left(1+\sum_{j=1}^{r-1} \sum_{1 \leq s_{1} \leq \cdots \leq s_{j} \leq n} \frac{\theta^{j}}{\left(\theta+s_{1}\right)\left(\theta+s_{2}\right) \cdots\left(\theta+s_{j}\right)}\right) .
\end{aligned}
$$

Proof Take $m=n, \beta=\theta$ in (10) of Theorem 4 , it is a must that $\theta \notin\{m+1, m+2, \ldots, n\}$, and define that $\prod_{k=n+1}^{n}=1, \sum_{i=1}^{0}=0$, then Corollary 6 follows.

Setting $r=0$ in (14), defining the empty sum $\sum_{j=1}^{-1}=-1$, we have the following familiar formula.

Corollary 7 For all $n \in \mathbb{N}$ and $\theta>0$,

$$
\sum_{k=0}^{n}(-1)^{k}\left(\begin{array}{l}
n \\
k
\end{array}\right)=0
$$

Setting $r=1$ in (14), defining the empty sum $\sum_{j=1}^{0}=0$, we have the following.

Corollary 8 (see [4]) For all $n \in \mathbb{N}$ and $\theta>0$,

$$
\sum_{k=0}^{n}(-1)^{k}\left(\begin{array}{l}
n \\
k
\end{array}\right) \frac{\theta}{\theta+k}=\prod_{k=1}^{n} \frac{k}{\theta+k}
$$

Setting $m=2$ in (14), we have the following.

Corollary 9 (see [4]) For all $n \in \mathbb{N}$ and $\theta>0$,

$$
\sum_{k=0}^{n}(-1)^{k}\left(\begin{array}{l}
n \\
k
\end{array}\right)\left(\frac{\theta}{\theta+k}\right)^{2}=\left(\prod_{k=1}^{n} \frac{k}{\theta+k}\right)\left(1+\sum_{k=1}^{n} \frac{\theta}{\theta+k}\right) .
$$

Corollary 10 Let $r, l, n \in \mathbb{N}$ and $\theta>0$. 
When $\theta \notin\{1,2, \ldots, n\}$,

$$
\begin{aligned}
\sum_{k=0}^{n}(-1)^{k}\left(\begin{array}{c}
n \\
k
\end{array}\right)\left(\begin{array}{c}
n+k \\
k
\end{array}\right)\left(\frac{\theta}{\theta+k}\right)^{r} \\
=\left(\prod_{k=1}^{n} \frac{k-\theta}{\theta+k}\right)\left(1+\sum_{j=1}^{r-1} \sum_{1 \leq s_{1} \leq \cdots \leq s_{j} \leq n} \frac{\theta^{j}}{\left(\theta+s_{1}\right)\left(\theta+s_{2}\right) \cdots\left(\theta+s_{j}\right)}\right. \\
\left.\quad+\sum_{i+l=r-1} \sum_{i=1}^{n} \sum_{1 \leq k_{1}<\cdots<k_{i} \leq n} \sum_{0 \leq s_{1} \leq \cdots \leq s_{l} \leq n} \frac{\theta^{r-1}}{\left(k_{1}-\theta\right) \cdots\left(k_{i}-\theta\right)\left(\theta+s_{1}\right) \cdots\left(\theta+s_{l}\right)}\right) .
\end{aligned}
$$

When $\theta \in\{1,2, \ldots, n\}$, we have

$$
\begin{aligned}
\sum_{k=0}^{n}(-1)^{k}\left(\begin{array}{c}
n \\
k
\end{array}\right)\left(\begin{array}{c}
n+k \\
k
\end{array}\right)\left(\frac{\theta}{\theta+k}\right)^{r} & \left(\prod_{k=1}^{n} \frac{1}{\theta+k}\right)\left(\prod_{k=1, k \neq \theta}^{n}(k-\theta)\right)\left(\theta+\sum_{j=1}^{r-2} \sum_{\substack{1 \leq s_{1} \leq \cdots \leq s_{j} \leq n\\
}} \frac{\theta^{j}}{\left(\theta+s_{1}\right)\left(\theta+s_{2}\right) \cdots\left(\theta+s_{j}\right)}\right. \\
& \left.+\sum_{i+l=r-2} \sum_{i=1}^{n-1} \sum_{\substack{k_{1}, \ldots, k_{i} \neq \theta \\
1 \leq k_{1}<\cdots<k_{i} \leq n}} \sum_{0 \leq s_{1} \leq \cdots \leq s_{l} \leq n} \frac{\theta^{r-1}}{\left(k_{1}-\theta\right) \cdots\left(k_{i}-\theta\right)\left(\theta+s_{1}\right) \cdots\left(\theta+s_{l}\right)}\right) .
\end{aligned}
$$

Proof Take $m=0, \beta=\theta$ in Theorem 4, then Corollary 10 follows.

Setting $r=0$ in (18) and defining the suitable empty sum, we have the following familiar formula.

Corollary 11 ([7]) For all $n \in \mathbb{N}$ and $\theta>0$,

$$
\sum_{k=0}^{n}(-1)^{k}\left(\begin{array}{l}
n \\
k
\end{array}\right)\left(\begin{array}{c}
n+k \\
k
\end{array}\right)=(-1)^{n}
$$

Setting $r=1$ and $\theta=\beta$ in (18) and defining the empty sum $\sum_{j=1}^{0}=0$, we have the following new binomial identity.

Corollary 12 For all $n \in \mathbb{N}$ and $\theta>0$,

$$
\sum_{k=0}^{n}(-1)^{k}\left(\begin{array}{c}
n \\
k
\end{array}\right)\left(\begin{array}{c}
n+k \\
k
\end{array}\right) \frac{\theta}{\theta+k}=\left(\prod_{k=1}^{n} \frac{k-\theta}{\theta+k}\right) .
$$

Setting $r=2$ and $\theta=\beta$ in (18) and defining the empty sum $\sum_{j=1}^{0}=0$, we have the following new binomial identity.

Corollary 13 For all $n \in \mathbb{N}$ and $\theta>0$,

$$
\sum_{k=0}^{n}(-1)^{k}\left(\begin{array}{l}
n \\
k
\end{array}\right)\left(\begin{array}{c}
n+k \\
k
\end{array}\right)\left(\frac{\theta}{\theta+k}\right)^{2}=\left(\prod_{k=1}^{n} \frac{k-\theta}{\theta+k}\right)\left(1+\sum_{k=1}^{n} \frac{\theta}{\theta+k}\right) .
$$


Theorem 14 For $r, l, n \in \mathbb{N}, 0 \leq m \leq n, \beta \neq 0$.

When $\theta \notin\{0,1,2, \ldots, n\}$, we have

$$
\begin{aligned}
\sum_{k=0}^{n}(-1)^{k}\left(\begin{array}{c}
n \\
k
\end{array}\right)\left(\begin{array}{c}
n+k \\
m+k
\end{array}\right)\left(\frac{\beta}{k-\theta}\right)^{r} & \\
= & (-1)^{n+1}\left(\begin{array}{c}
\theta-1 \\
n
\end{array}\right)^{-1}\left(\begin{array}{c}
n+\theta \\
m+\theta
\end{array}\right)\left(-\left(-\frac{\beta}{\theta}\right)^{r}+\sum_{j=1}^{r-1} \sum_{1 \leq s_{1} \leq \cdots \leq s_{j} \leq n} \frac{\beta^{r}}{\theta\left(s_{1}-\theta\right) \cdots\left(s_{j}-\theta\right)}\right. \\
& \left.+\sum_{i+l=r-1} \sum_{i=1}^{n-m} \sum_{m+1 \leq k_{1}<\cdots<k_{i} \leq n} \sum_{0 \leq s_{1} \leq \cdots \leq s_{l} \leq n} \frac{\beta^{r}}{\theta\left(k_{1}+\theta\right) \cdots\left(k_{i}+\theta\right)\left(s_{1}-\theta\right) \cdots\left(s_{l}-\theta\right)}\right) .
\end{aligned}
$$

When $\theta \in\{0,1,2, \ldots, n\}$, we have

$$
\begin{aligned}
\sum_{k=0, k \neq \theta}^{n}(-1)^{k}\left(\begin{array}{l}
n \\
k
\end{array}\right)\left(\begin{array}{c}
n+k \\
m+k
\end{array}\right)\left(\frac{\beta}{k-\theta}\right)^{r} \\
=(-1)^{\theta+1}\left(\begin{array}{l}
n \\
\theta
\end{array}\right)\left(\begin{array}{c}
n+\theta \\
m+\theta
\end{array}\right)\left(\left(-\frac{\beta}{\theta}\right)^{r}+\sum_{j=1}^{r} \sum_{\substack{s_{1}, \ldots, s_{j} \neq \theta \\
1 \leq s_{1} \leq \cdots \leq s_{j} \leq n}} \frac{\beta^{r}}{\left(s_{1}-\theta\right) \cdots\left(s_{j}-\theta\right)}\right. \\
\left.\quad+\sum_{i+l=r} \sum_{i=1}^{n-m} \sum_{m+1 \leq k_{1}<\cdots<k_{i} \leq n} \sum_{\substack{s_{1}, \ldots, s_{l} \neq \theta \\
0 \leq s_{1} \leq \cdots \leq s_{l} \leq n}} \frac{\beta^{r}}{\left(k_{1}+\theta\right) \cdots\left(k_{i}+\theta\right)\left(s_{1}-\theta\right) \cdots\left(s_{l}-\theta\right)}\right) .
\end{aligned}
$$

Proof By means of Theorem 1 and the residues calculus, we obtain the following.

- When $\theta \notin\{0,1,2, \ldots, n\}$, taking $f(z)=\left(\frac{\beta}{z-\theta}\right)^{r}$ in $(8)$, and $\mathcal{C}$ is a positively oriented, encloses the poles $0,1,2, \ldots, n$ and no others.

$$
\begin{aligned}
\sum_{k=0}^{n} & (-1)^{k}\left(\begin{array}{l}
n \\
k
\end{array}\right)\left(\begin{array}{c}
n+k \\
m+k
\end{array}\right)\left(\frac{\beta}{k-\theta}\right)^{r} \\
& =\frac{(-1)^{n} n !}{2 \pi i(n-m) !} \int_{\mathcal{C}} \frac{(m+z+1)_{n-m}}{z(z-1) \cdots(z-n)}\left(\frac{\beta}{z-\theta}\right)^{r} d z \\
& =\frac{(-1)^{n+1} n !}{(n-m) !} \operatorname{Res} \frac{(m+z+1)_{n-m}}{z=\theta}\left(\frac{\beta}{z-\theta}\right)^{r} \\
& =\frac{(-1)^{n+1} n !}{(n-m) !} \operatorname{Res}_{z=\theta} \frac{(m+z+1)_{n-m}}{z(z-1) \cdots(z-n)} \frac{\beta^{r}}{(z-\theta)^{r}} \\
& =\frac{(-1)^{n+1} n !}{(n-m) !}\left[(z-\theta)^{r-1}\right] \frac{(z+1+m)(z+2+m) \cdots(z+n) \beta^{r}}{z(z-1) \cdots(z-n)} \\
& =\frac{(-1)^{n+1} n !}{(n-m) !}\left[z^{r-1}\right] \frac{(z+1+m+\theta)(z+2+m+\theta) \cdots(z+n+\theta) \beta^{r}}{(z+\theta)(z+\theta-1) \cdots(z+\theta-n)} \\
& =(-1)^{n+1} \frac{n !(\theta-n-1) !(m+1+\theta)_{n-m}}{(n-m) ! \theta !}\left[z^{r-1}\right] \frac{\left(1+\frac{z}{m+1+\theta}\right) \cdots\left(1+\frac{z}{n+\theta}\right) \beta^{r}}{\left(1+\frac{z}{\theta}\right)\left(1+\frac{z}{\theta-1}\right) \cdots\left(1+\frac{z}{\theta-n}\right)} \\
& =(-1)^{n+1}\left(\begin{array}{c}
\theta-1 \\
n
\end{array}\right)\left(\begin{array}{c}
n+\theta \\
m+\theta
\end{array}\right)\left[z^{r-l-1}\right]\left(1+\sum_{i=1}^{n-m} \sum_{m+1 \leq k_{1}<\cdots<k_{i} \leq n} \frac{z^{i}}{\left(k_{1}+\theta\right) \cdots\left(k_{i}+\theta\right)}\right)
\end{aligned}
$$




$$
\begin{aligned}
& \times \sum_{0 \leq s_{1} \leq \cdots \leq s_{l} \leq n} \frac{\beta^{r}}{\theta\left(s_{1}-\theta\right) \cdots\left(s_{l}-\theta\right)} \\
= & (-1)^{n+1}\left(\begin{array}{c}
\theta-1 \\
n
\end{array}\right)^{-1}\left(\begin{array}{c}
n+\theta \\
m+\theta
\end{array}\right)\left[z^{r-l-1}\right]\left(1+\sum_{i=1}^{n-m} \sum_{m+1 \leq k_{1}<\cdots<k_{i} \leq n} \frac{z^{i}}{\left(k_{1}+\theta\right) \cdots\left(k_{i}+\theta\right)}\right) \\
& \times\left((-1)^{l} \frac{\beta^{r}}{\theta^{l+1}}+\sum_{j=1}^{l} \sum_{1 \leq s_{1} \leq \cdots \leq s_{j} \leq n} \frac{\beta^{r}}{\theta\left(s_{1}-\theta\right) \cdots\left(s_{j}-\theta\right)}\right) \\
= & (-1)^{n+1}\left(\begin{array}{c}
\theta-1 \\
n
\end{array}\right)^{-1}\left(\begin{array}{c}
n+\theta \\
m+\theta
\end{array}\right)\left(-\left(-\frac{\beta}{\theta}\right)^{r}+\sum_{j=1}^{r-1} \sum_{1 \leq s_{1} \leq \cdots \leq s_{j} \leq n} \frac{\beta^{r}}{\theta\left(s_{1}-\theta\right) \cdots\left(s_{j}-\theta\right)}\right. \\
& \left.+\sum_{i+l=r-1} \sum_{i=1}^{n-m} \sum_{m+1 \leq k_{1}<\cdots<k_{i} \leq n} \sum_{0 \leq s_{1} \leq \cdots \leq s_{l} \leq n} \frac{\beta^{r}}{\theta\left(k_{1}+\theta\right) \cdots\left(k_{i}+\theta\right)\left(s_{1}-\theta\right) \cdots\left(s_{l}-\theta\right)}\right) .
\end{aligned}
$$

- When $\theta \in\{0,1,2, \ldots, n\}$, taking $f(z)=\left(\frac{\beta}{z-\theta}\right)^{r}$ in $(8)$, and $\mathcal{C}$ is a positively oriented, encloses the poles $0,1, \ldots, \theta-1, \theta+1, \ldots, n$ and no others.

$$
\begin{aligned}
& \sum_{k=0, k \neq \theta}^{n}(-1)^{k}\left(\begin{array}{l}
n \\
k
\end{array}\right)\left(\begin{array}{c}
n+k \\
m+k
\end{array}\right)\left(\frac{\beta}{k-\theta}\right)^{r} \\
& =\frac{(-1)^{n} n !}{2 \pi i(n-m) !} \int_{\mathcal{C}} \frac{(m+z+1)_{n-m}}{z(z-1) \cdots(z-n)}\left(\frac{\beta}{z-\theta}\right)^{r} d z \\
& =\frac{(-1)^{n+1} n !}{(n-m) !} \operatorname{Res}_{z=\theta} \frac{(m+z+1)_{n-m}}{z(z-1) \cdots(z-n)}\left(\frac{\beta}{z-\theta}\right)^{r} \\
& =\frac{(-1)^{n+1} n !}{(n-m) !} \operatorname{Res}_{z=\theta} \frac{(m+z+1)_{n-m}}{z(z-1) \cdots(z-\theta+1)(z-\theta-1) \cdots(z-n)} \frac{\beta^{r}}{(z-\theta)^{r+1}} \\
& =\frac{(-1)^{n+1} n !}{(n-m) !}\left[(z-\theta)^{r}\right] \frac{(z+1+m)(z+2+m) \cdots(z+n) \beta^{r}}{z(z-1) \cdots(z-\theta+1)(z-\theta-1) \cdots(z-n)} \\
& =\frac{(-1)^{n+1} n !}{(n-m) !}\left[z^{r}\right] \frac{(z+1+m+\theta)(z+2+m+\theta) \cdots(z+n+\theta) \beta^{r}}{(z+\theta)(z+\theta-1) \cdots(z+1)(z-1) \cdots(z+\theta-n)} \\
& =(-1)^{\theta+1} \frac{n !(m+1+\theta)_{n-m}}{(n-m) ! \theta !(n-\theta) !}\left[z^{r}\right] \frac{\left(1+\frac{z}{m+1+\theta}\right) \cdots\left(1+\frac{z}{n+\theta}\right) \beta^{r}}{\left(1+\frac{z}{\theta}\right)\left(1+\frac{z}{\theta-1}\right) \cdots(1+z)(1-z) \cdots\left(1-\frac{z}{n-\theta}\right)} \\
& =(-1)^{\theta+1}\left(\begin{array}{c}
n \\
\theta
\end{array}\right)\left(\begin{array}{c}
n+\theta \\
m+\theta
\end{array}\right)\left[z^{r-l}\right]\left(1+\sum_{i=1}^{n-m} \sum_{m+1 \leq k_{1}<\cdots<k_{i} \leq n} \frac{z^{i}}{\left(k_{1}+\theta\right) \cdots\left(k_{i}+\theta\right)}\right) \\
& \times \sum_{\substack{s_{1}, \ldots, s_{l} \neq \theta \\
0 \leq s_{1} \leq \cdots \leq s_{l} \leq n}} \frac{\beta^{r}}{\left(s_{1}-\theta\right) \cdots\left(s_{l}-\theta\right)} \\
& =(-1)^{\theta+1}\left(\begin{array}{l}
n \\
\theta
\end{array}\right)\left(\begin{array}{c}
n+\theta \\
m+\theta
\end{array}\right)\left[z^{r-l}\right]\left(1+\sum_{i=1}^{n-m} \sum_{m+1 \leq k_{1}<\cdots<k_{i} \leq n} \frac{z^{i}}{\left(k_{1}+\theta\right) \cdots\left(k_{i}+\theta\right)}\right) \\
& \times\left(\frac{\beta^{r}}{(-\theta)^{l}}+\sum_{j=1}^{l} \sum_{\substack{s_{1}, \ldots, s_{j} \neq \theta \\
1 \leq s_{1} \leq \cdots \leq s_{j} \leq n}} \frac{\beta^{r}}{\left(s_{1}-\theta\right) \cdots\left(s_{j}-\theta\right)}\right)
\end{aligned}
$$




$$
\begin{aligned}
= & (-1)^{\theta+1}\left(\begin{array}{c}
n \\
\theta
\end{array}\right)\left(\begin{array}{c}
n+\theta \\
m+\theta
\end{array}\right)\left(\left(-\frac{\beta}{\theta}\right)^{r}+\sum_{j=1}^{r} \sum_{\substack{s_{1}, \ldots, s_{j} \neq \theta \\
1 \leq s_{1} \leq \cdots \leq s_{j} \leq n}} \frac{\beta^{r}}{\left(s_{1}-\theta\right) \cdots\left(s_{j}-\theta\right)}\right. \\
& \left.+\sum_{i+l=r} \sum_{i=1}^{n-m} \sum_{m+1 \leq k_{1}<\cdots<k_{i} \leq n} \sum_{\substack{s_{1} \leq s_{1} \leq \cdots \leq s_{l} \leq n \\
s_{1}, \ldots, s_{l} \neq \theta}} \frac{\beta^{r}}{\left(k_{1}+\theta\right) \cdots\left(k_{i}+\theta\right)\left(s_{1}-\theta\right) \cdots\left(s_{l}-\theta\right)}\right) .
\end{aligned}
$$

This proof is complete.

Corollary 15 For $r, n \in \mathbb{N}$.

When $\theta \notin\{0,1,2, \ldots, n\}$, we have

$$
\begin{aligned}
& \sum_{k=0}^{n}(-1)^{k}\left(\begin{array}{l}
n \\
k
\end{array}\right)\left(\frac{\theta}{k-\theta}\right)^{r} \\
& \quad=(-1)^{n}\left(\begin{array}{c}
\theta-1 \\
n
\end{array}\right)^{-1}\left(1-\sum_{j=1}^{r-1} \sum_{1 \leq s_{1} \leq \cdots \leq s_{j} \leq n} \frac{\theta^{j}}{\left(s_{1}-\theta\right) \cdots\left(s_{j}-\theta\right)}\right) .
\end{aligned}
$$

When $\theta \in\{0,1,2, \ldots, n\}$, we have

$$
\begin{aligned}
& \sum_{k=0, k \neq \theta}^{n}(-1)^{k}\left(\begin{array}{l}
n \\
k
\end{array}\right)\left(\frac{\theta}{k-\theta}\right)^{r} \\
& =(-1)^{\theta+1}\left(\begin{array}{l}
n \\
\theta
\end{array}\right)\left((-1)^{r}+\sum_{j=1}^{r} \sum_{\substack{s_{1}, \ldots, s_{j} \neq \theta \\
1 \leq s_{1} \leq \cdots \leq s_{j} \leq n}} \frac{\theta^{j}}{\left(s_{1}-\theta\right) \cdots\left(s_{j}-\theta\right)}\right) .
\end{aligned}
$$

Proof Take $m=n, \beta=\theta$ in Theorem 14 and define that $\prod_{k=n+1}^{n}=1, \sum_{i=1}^{0}=0$, then Corollary 15 follows.

Corollary 16 For $r, l, n \in \mathbb{N}$.

When $\theta \notin\{0,1,2, \ldots, n\}$, we have

$$
\begin{aligned}
& \sum_{k=0}^{n}(-1)^{k}\left(\begin{array}{l}
n \\
k
\end{array}\right)\left(\begin{array}{c}
n+k \\
k
\end{array}\right)\left(\frac{\theta}{k-\theta}\right)^{r} \\
& =(-1)^{n+1}\left(\begin{array}{c}
\theta-1 \\
n
\end{array}\right)^{-1}\left(\begin{array}{c}
n+\theta \\
\theta
\end{array}\right)\left((-1)^{r+1}+\sum_{j=1}^{r-1} \sum_{1 \leq s_{1} \leq \cdots \leq s_{j} \leq n} \frac{\theta^{j}}{\left(s_{1}-\theta\right) \cdots\left(s_{j}-\theta\right)}\right. \\
& \left.\quad+\sum_{i+l=r-1} \sum_{i=1}^{n} \sum_{1 \leq k_{1}<\cdots<k_{i} \leq n} \sum_{0 \leq s_{1} \leq \cdots \leq s_{l} \leq n} \frac{\theta^{r-1}}{\left(k_{1}+\theta\right) \cdots\left(k_{i}+\theta\right)\left(s_{1}-\theta\right) \cdots\left(s_{l}-\theta\right)}\right) .
\end{aligned}
$$

When $\theta \in\{0,1,2, \ldots, n\}$, we have

$$
\begin{aligned}
& \sum_{k=0, k \neq \theta}^{n}(-1)^{k}\left(\begin{array}{l}
n \\
k
\end{array}\right)\left(\begin{array}{c}
n+k \\
k
\end{array}\right)\left(\frac{\theta}{k-\theta}\right)^{r} \\
& =(-1)^{\theta+1}\left(\begin{array}{l}
n \\
\theta
\end{array}\right)\left(\begin{array}{c}
n+\theta \\
\theta
\end{array}\right)
\end{aligned}
$$




$$
\begin{aligned}
& \times\left((-1)^{r}+\sum_{j=1}^{r} \sum_{\substack{s_{1}, \ldots, s_{j} \neq \theta \\
1 \leq s_{1} \leq \cdots \leq s_{j} \leq n}} \frac{\theta^{j}}{\left(s_{1}-\theta\right) \cdots\left(s_{j}-\theta\right)}\right. \\
& \left.+\sum_{i+l=r} \sum_{i=1}^{n} \sum_{1 \leq k_{1}<\cdots<k_{i} \leq n} \sum_{\substack{s_{1}, \ldots, s_{l} \neq \theta \\
0 \leq s_{1} \leq \cdots \leq s_{l} \leq n \\
0}} \frac{\theta^{r}}{\left(k_{1}+\theta\right) \cdots\left(k_{i}+\theta\right)\left(s_{1}-\theta\right) \cdots\left(s_{l}-\theta\right)}\right)
\end{aligned}
$$

Proof Take $m=0, \beta=\theta$ in Theorem 14 and define that $\prod_{k=n+1}^{n}=1, \sum_{i=1}^{0}=0$, then Corollary 16 follows.

Remark 17 By applying Bell polynomials (3) and (4), we can deduce all the results of [18] and [19]. Our results are another representation of the corresponding results in [18] and [19]. For example, compare formula (24) with $m=0, \theta=M, \beta=1$ and the following formula [19]: For $0 \leq M \leq n$,

$$
\begin{aligned}
& \sum_{0 \leq k \leq n, k \neq M}(-1)^{k-1}\left(\begin{array}{c}
n \\
k
\end{array}\right)\left(\begin{array}{c}
n+k \\
k
\end{array}\right) \frac{1}{(k-M)^{r}} \\
= & (-1)^{M}\left(\begin{array}{c}
n \\
M
\end{array}\right)\left(\begin{array}{c}
M+n \\
M
\end{array}\right) \sum_{m_{1}+2 m_{2}+3 m_{3}+\cdots=r} \frac{1}{m_{1} ! m_{2} ! m_{3} ! \cdots}\left(\frac{x_{1}}{1}\right)^{m_{1}}\left(\frac{x_{2}}{2}\right)^{m_{2}}\left(\frac{x_{3}}{3}\right)^{m_{3}} \cdots,
\end{aligned}
$$

where

$$
x_{k}=(-1)^{k-1} H_{M+n}^{(k)}-2 H_{M}^{(k)}+H_{n-M}^{(k)}, \quad H_{n}^{(r)}=\sum_{k=1}^{n} \frac{1}{k^{r}}, n, r \in \mathbb{N} .
$$

Obviously, formula (24) includes the above formula (29) and the main result of [18], i.e., formula (24) is an extension of them.

\section{Competing interests}

The authors declare that they have no competing interests.

\section{Authors' contributions}

All authors contributed equally in writing this paper, and read and approved the manuscript.

\section{Acknowledgements}

The authors express their sincere gratitude to referee for many valuable comments and suggestions. The present investigation was supported by Natural Science Foundation Project of Chongqing, China under Grant CSTC2011 JJA00024, Research Project of Science and Technology of Chongqing Education Commission, China under Grant KJ120625, Fund of Chongaing Normal University, China under Grant 10XLR017 and 2011XLZ07.

Received: 17 September 2014 Accepted: 13 January 2015 Published online: 27 January 2015

\section{References}

1. Comtet, L: Advanced Combinatorics: The Art of Finite and Infinite Expansions. Reidel, Dordrecht (1974)

2. Flajolet, P, Sedgewick, R: Mellin transforms and asymptotics: finite differences and Rice's integrals. Theor. Comput. Sci. 144, 101-124 (1995)

3. Nörlund, NE: Vorlesungen über Differentzenrechnung. Springer, Berlin (1924); Reprinted by Chelsea Publishing Company, Bronx, New York (1954)

4. Jonathon, P: A probabilistic proof of a binomial identity. Am. Math. Mon. 120, 558-562 (2013)

5. Bayad, A, Simsek, Y, Srivastava, HM: Some array type polynomials associated with special numbers and polynomials. Appl. Math. Comput. 244, 149-157 (2014) 
6. Simsek, Y: Analysis of the Bernstein basis functions: an approach to combinatorial sums involving binomial coefficients and Catalan number. Math. Methods Appl. Sci. (2014). doi:10.1002/mma.327

7. Gould, HW: Combinatorial Identities: A Standardized Set of Tables Listing 500 Binomial Coefficient Summations, viii+106 pp. Henry W. Gould, Morgantown (1972)

8. Liu, H, Wang, W: Harmonic number identities via hypergeometric series and Bell polynomials. Integral Transforms Spec. Funct. 23, 49-68 (2012)

9. Mansour, T: Identities on harmonic and q-harmonic number sums. Afr. Math. 23, 135-143 (2012)

10. Prodinger, H: Human proofs of identities by Osburn and Schneider. Integers 8, \#A10 (2008)

11. Prodinger, H: Mortenson's identities and partial fraction decomposition. Util. Math. (in press). http://math.sun.ac.za/ hproding/paperlst.htm

12. Tauraso, R, Prodinger, H: New multiple harmonic sum identities. Electron. J. Comb. 21, \#P2.43 (2014)

13. Sofo, A: Sums of derivatives of binomial coefficients. Adv. Appl. Math. 42, 123-134 (2009)

14. Spieß, J: Some identities involving harmonic numbers. Math. Comput. 55, 839-863 (1990)

15. Wang, W: Riordan arrays and harmonic number identities. Comput. Math. Appl. 60, 1494-1509 (2010)

16. Yan, Q, Wei, C, Fan, X: Q-Generalizations of Mortenson's identities and further identities. Ramanujan J. (2014). doi:10.1007/s11139-013-9526-7

17. Zhang, ZZ, Wang, J: A common generalization of some identities. Integers 5, \#A33 (2005)

18. Kirschenhofer, P: A note on alternating sums. Electron. J. Comb. 3, \#R7 (1996)

19. Prodinger, H: Identities involving harmonic numbers that are of interest for physicists. Util. Math. 83, 291-299 (2010)

\section{Submit your manuscript to a SpringerOpen ${ }^{\circ}$ journal and benefit from:}

- Convenient online submission

- Rigorous peer review

- Immediate publication on acceptance

Open access: articles freely available online

High visibility within the field

- Retaining the copyright to your article 